\title{
Fatigue equivalent load approach for fatigue design
}

\author{
Ida Raoult ${ }^{1, *}$ and Benoit Delattre ${ }^{1, * *}$ \\ ${ }^{1}$ Science and Future Technologies Department, Groupe PSA - Centre Technique de Vélizy, Route de \\ Gisy, Parc Inovel Sud, 78943 Vélizy-Villacoublay Cedex, France
}

\begin{abstract}
Industrial structures undergo complex loading often unsuitable for the design and validation phases of their development. Engineers seek simplified signals to replace them, yet equivalent in terms of fatigue. A general framework for the construction of fatigue equivalent loads is presented, intended to take into account some uncertainty on the structure to which it will apply. The guiding idea is to describe this uncertainty thanks to parameters of a model of the structure, and then to assure the equivalence of damage or failure behavior over the whole range of expected parameters. The approach is applied in the case of structures undergoing multiple inputs of concentrated force.
\end{abstract}

\section{Introduction}

\subsection{Motivation}

Engineers wish to design a mechanical structure against fatigue so that it ensures its function for a given time and in most use cases. Here, the structure is considered defective as soon as a crack initiates, even if, in practice, it can often continue to fulfill its function with a crack. The choice of an initiation failure criterion is a conservative choice, often made in the automotive industry, because there is no obligatory maintenance in the automotive field except for the legal vehicle inspection. It also greatly simplifies the simulations since the geometry of the components does not change during its lifetime.

Loads on the structure can be measured directly in service, or in conditions representative of the use. In the case of a car component, a vehicle is equipped with multiple sensors and then driven on a test track. The forces applying to the component of interest are recorded; there are usually several channels of them, either that the part has several interfaces with the rest of the structure, or that there are several distinct components of forces, acting on different directions, at these interfaces (see 1). The resulting record is then used as a design requirement. The structure must withstand this sequence of forces, repeated as many times as needed to guaranty its target strength. This target behavior can be determined by the stressstrength interference method (see the writings of Lemaire [1] for example).

The strength of the components is estimated by simulation and proven by the test. Unfortunately, the previously mentioned recording is generally unsuitable for both, because of its complexity and duration.

\footnotetext{
*e-mail: ida.raoult@mpsa.com

**e-mail: benoit.delattre@mpsa.com
} 
First, consider the case of simulation. When the structure of interest responds linearly (linear elasticity in small deformations, absence of contact, etc.), a reduced number of simulations, corresponding to the unit response of the structure under each independent degree of freedom of the loading (for each force channel) allows to reconstruct the solution under the complete loading by linear combination. When the structure has a non-linear response, like a car suspension, this operation is no longer possible and each instant of the signal must be calculated. In practice, the necessary computation times are excessively long.

The tests present even worse difficulties. First, the signal is difficult to reproduce on a test bench. Each channel must be controlled correctly regardless of the value of the other channels, a difficult task since each structure exhibits its own couplings. Moreover, the signal can contain high frequencies, in limit of capability of the hydraulic cylinders. Secondly, the signal is long (usually, several weeks) in comparison with the typical times of a development project. When the part response is independent of the strain rate, it is possible to accelerate the signal by a simple compression of the time base, provided that the machines are capable of it. For large structures, the loading rate cannot be increased significantly. The duration and complexity of the tests usually imply high costs.

In both cases, it is desired to have a simpler loading, but nevertheless equivalent - in the sense of the strength criterion - to the original signal. Let us call it the equivalent signal. The objective of this paper is to propose a general framework for constructing these equivalent signals.

Note that even if this work was performed in the framework of the automotive industry, the question addressed here is of interest for many others, especially in the transportation area [2] but not only [3].

\subsection{A poorly known structure}

When the structure is fully designed, the damage under the original signal may be assessed by simulation, using some damage model. The process can be repeated with any other other simplified signal until one induces the same damage. This is a procedure used for example for the validation of wind turbines [4]. However this method is not applicable in many cases. Firstly, the calculations may be excessively long, especially when the structure exhibits a non linear behavior. Secondly, the imperfection of models, in terms of geometry as well as material behaviors, or uncertainty on some parameters, may induce a significant error [4]. This error can be reduced by multiplying the simulations, so as to take into account a variety of cases, but then again, the cost of simulation might be too high. To workaround the calculation time problem, it is possible to replace the detailed model by a simplified one, usually called meta-model [5].

Nonetheless, even those sophisticated methods cannot solve the main problem encountered in many design projects : when the validation test signal is required, the component is not designed yet, or only in an early version. This situation happens, in particular, when the component is bought from a supplier. The signal to withstand has to be known in advance; it is part of the specification. Even in a fully internal design process, the first stages of design need simplified specifications, robust to small variations of geometry.

The equivalent signal is usually described by few parameters. Those parameters are easy to compare from one project to the other, which helps classifying technical solutions. Moreover, the distribution of these parameters for a given application can be used for building specification standards (very useful in the first stages of design). The parameters of the equivalent signal can also be used for reliable design as a stress variable in the stress-strength interference method [1]. 


\subsection{Fatigue equivalent approach in the literature}

\subsubsection{Elastic quasi-static structure, $1 D$ load}

The fatigue equivalent approach is mainly used with elastic structures undergoing a quasistatic 1D concentrated force $F$. The loading is $1 \mathrm{D}$ in the sens that it is described by a single scalar, but varies over time, in a variable amplitude fashion. Then, the stress at some time $t$ and point $M$ writes simply as :

$$
\sigma(M, t)=\sigma_{L}(M) F(t)
$$

with $\sigma_{L}$ the local stress experienced at point $\mathrm{M}$ for a unit force $F$. The material is given and we assume that its $S N$ curve can be simply modeled with the Basquin law :

$$
N=\left(\frac{\sigma_{a}}{\sigma_{1}}\right)^{-\beta}
$$

with $\sigma_{1}$ and $\beta$ some known material parameters and $\sigma_{a}$ the amplitude of the principal stress (or any invariant of the local stress tensor). The sequence effect is ignored. The force signal is decomposed into unit cycles using the Rainflow procedure [6]. Since the invariant is a homogeneous function of the stress tensor (i.e. with a multiplicative scaling behavior), the cycles of the local variable responsible for damage (here, the principal stress) are just scaled from the force cycles and their amplitude is:

$$
\sigma_{a}(M, j)=\sigma_{L a}(M) F_{a}(j)
$$

with $j$ the index of the Rainflow cycle. The linear Palmgren-Miner accumulation law gives the local damage $d$ such as:

$$
d(M)=\left(\frac{\sigma_{L}(M)}{\sigma_{1}}\right)^{\beta} \sum_{j} F_{a}^{\beta}(j)
$$

A fatigue damage equivalent signal is searched in the form of a constant amplitude signal with a given number of cycles $N_{e q}$ (usually close to the apparent endurance limit or kneepoint of the $S N$ curve). The signal is said to be equivalent if it yields the same damage as the original signal :

$$
\forall M, \quad N_{e q}\left(\frac{\sigma_{L}(M)}{\sigma_{1}}\right)^{\beta} F_{a e q}^{\beta}=\left(\frac{\sigma_{L}(M)}{\sigma_{1}}\right)^{\beta} \sum_{j} F_{a}^{\beta}(j)
$$

In a very opportune way, the localization factor $\sigma_{L}(M)$ and the Basquin constant $\sigma_{1}$ vanish, so that the condition can indeed be satisfied for every point of the structure with a unique $F_{a e q}$, given that the exponent $\beta$ is known :

$$
F_{a e q}=\left(\frac{1}{N_{e q}} \sum_{j} F_{a}^{\beta}(j)\right)^{\frac{1}{\beta}} .
$$

A mean stress correction such as Goodman's or Gerber's can easily be introduced in these equations, but an additional information on the structure will be required : the ultimate force $F_{u}$ of the structure will have to be given. It cannot be simply deduced from the material parameter $\sigma_{u}$ because in this case the localization factor does not vanish. The equivalent signal can then also have an additional parameter since its mean value influences its damage. 
Problems arise when the exponent $\beta$ (or $F_{u}$ ) is somewhat uncertain or known to be multiple (in the case when the structure is composed of several materials or suffers from several damage mechanisms). Each $\beta$ will then yield a different $F_{a e q}$. Many authors have performed some sensitivity study of the fatigue equivalent to material parameters (see [7] for example) to conclude that is was significant.

Thomas [8] proposes a method to circumvent the problem of the mean stress correction. The localization factor is supposed to be such that the structure fails exactly at the end of the original signal $\left(\sigma_{L}, d=1\right)$. Thus, the fatigue equivalence is realized for the structures of interest : the ones that might fail.

Freebury and Musial [4] propose to choose the parameters of the equivalent load so as to minimize its sensitivity to the uncertain parameters (Basquin's exponent and ultimate load of the structure), estimated on the whole range of possible parameters for their wind turbine blades application. This solution is not completely satisfying, because some error will remain; however it is possible to quantify this error on the range of uncertain parameters.

\subsubsection{Elastic vibrating structure, $1 D$ load}

The case of an elastic structure expected to vibrate under some 1D load is also extensively documented, especially for accelerated vibration testing (see for example [9]). The approach exposed above no longer holds because the localization stress is no longer independent of time. It depends on the dynamic response of the structure. To solve the problem, Lalanne [10] uses an approximation of the typical response of the structure: it is assumed to behave like a mass-spring system with a given damping coefficient and some unknown natural frequency, but on a given frequency range. Thus, for each frequency $f$ of this range, the local stress is proportional to the force signal filtered by the corresponding mass-spring system. The above equivalence method (without any mean stress correction) can then be applied frequency by frequency and the so-called "Fatigue Damage Spectrum" is built. A Gaussian synthetic signal can be found with the appropriate spectral content to ensure damage equivalence over a whole frequency range. It should be noted that:

- the behavior of the structure is approximated; the quality of the approximation should be verified a posteriori;

- the equivalence is given on a selected frequency range; it should also be checked that it encompasses the working space of the component in service;

- The frequency space is discrete. Indeed, each considered frequency gives an equation for the synthetic signal to satisfy. It must then have as many degrees of freedom (parameters) to calibrate.

This method is an improvement over the quasi-static case with unknown parameters since the equivalence is ensured for a whole range of the unknown parameter (the natural frequency), the cost of it being a high number of parameters for the equivalent signal (the power spectral density).

\subsubsection{Non-linear behavior, $1 D$ load}

When the structure exhibits a non linear behavior, the determination of a localization factor can dangerously impair the equivalent load approach. However, it is sometimes possible to approximate the response of a family of similar components. Szmytka [11] describes this approach for cylinder heads made of aluminum and subject to thermomecanical fatigue. The load is a thermal flux and the local damage a dissipated energy density. In this case, 
failure always occurs at the same location and the damage at this point can be obtained from simulations on multiple components, previously designed. This approach strongly relies on the perpetuation of the design of the component. Once the component is designed, the validity of the approximate response should be checked.

\subsubsection{Elastic quasi-static, $n D$ load}

The case of components subjected to multiple inputs of loads is considered as difficult (see [12], Chapter 6), even in the simple case where the linear superposition principle holds, because of the unknown interactions of the different loads at the local scale. Industrial practice is not well documented but to our knowledge usually consists in analyzing each channel independently. Sometimes the average correlation is taken into account by a transformation in the principal directions of the $\left(F_{i}\right)_{i}$.

In 2006, Genêt [13] establishes the first basis for a multi-input equivalent approach in the case of an elastic structure undergoing a finite number of forces $\left(F_{i}\right)_{i}$. A major assumption is made about the local behavior of the structure under these loads : each force $F_{i}$ induces at the potential critical point the same form of stress tensor :

$$
\sigma(M, t)=\sigma_{L}(M) C(M) \sum_{i} a_{i}(M) F_{i}(t)
$$

with $\sigma_{L}$ a unit localization tensor (i.e. $\left\|\sigma_{L}\right\|=1$ for a given norm), $\left(a_{i}\right)_{i}$ a unit vector (i.e. such that $\left\|\left(a_{i}\right) i\right\|=1$ ) of coefficients giving the relative importance, locally, of each input force $F_{i}$, and $C$ an average intensity factor. Then, using either a coarse damage model (such as Basquin's model) or a more sophisticated one (a simplified version of Morel's model [14]), the damage of the original signal and that of an equivalent signal can be written. When equating those two damages, the localization stress tensor $\sigma_{L}$ vanishes, so that the only point $(M)$ dependent terms remaining in the equation are the $C$ and $a_{i}$. The author then proposes to browse the $a_{i}$ (i.e. the different possible combinations of $F_{i}$ ) and to look for a fatigue equivalent loading such that its damage would be as close as possible to the damage induced by the original signal for all the $a_{i}$. In the case of Basquin's model, the intensity factor $C$ vanishes (as in the 1D case described in paragraph 1.3.1) but it is not the case for Morel's criterion. There, the model describes a fatigue limit, so that the intensity factor $C$ greatly impacts the calculated damage by selecting the threshold above which the signal induces damage. The author then proposes to reduce the damage equivalence to special structures of interest, with $C$ factors such that the damage of the original signal is exactly one.

This interesting approach was not given much more attention in the following years, because the basic assumption of proportional stresses (equation 7) was deemed too strong for most industrial applications. A close look at critical points on many structures yet often reveals notched areas which strongly orient the local stress, indicating a possible application of the method. Anyway, this hypothesis can be relaxed, as will be seen further in this paper.

Recently, Roux[15] proposed an alternative to this hypothesis. The equivalence is seeked for every points of a finite number of similar structures already in use, here, train wheels. The local stress tensors are taken at each integration point of a finite element model of each wheel. He shows that the equivalent signal obtained for each wheel is very similar, and can serve as a base for a specification test for future wheels. The advantage of this method is that is takes into account the effective response of the structure, but the major drawback is that it applies only to a family of structures with very similar geometries, excluding innovative design. An improvement of the method could be to precise the scope of application of the method, using parameterized geometries in the library of structures used for determining the fatigue equivalent load. 


\section{Formalization of the approach}

The method described below is a generalization of the one exposed in [16] in the case of an elastic structure.

\subsection{Position of the problem}

Consider a structure, submitted to a finite (usually small) number $n_{F}$ of global loads $\mathrm{F}=$ $\left(F_{i}\right)_{i \in\left[1, n_{F}\right]}$. These global loads are usually forces, hence the choice of notation, but can be any kind of load such as displacement, acceleration or imposed temperature, applied on the structure at a global scale. These loads vary on a given period of time $T$ and are measured on a finite number $n_{T}$ of instants.

\subsection{Structural damage model}

The structure $\Omega$ fails if it does so in at least one location. A damage variable $d$ is evaluated in each point $M$. Failure happens when $\exists M, d(M)=1$. A model is needed to relate the damage in each point to the loading undergone by the structure. This model is usually composed of two elements : a localization function and a material damage function.

The localization function $L$ associates, for each point $M$, the history of global loads $\mathrm{F}(t), t \in[0, T]$ and structural parameters $p_{L}$ to the history of local variables $\sigma(t), t \in[0, T]$.

$$
\left[\begin{array}{c}
(\mathrm{F}(t))_{t \in[0, T]} \\
p_{L}(M), M \in \Omega
\end{array}\right] \stackrel{L}{\longrightarrow}(\sigma(t))_{t \in[0, T]}
$$

These local variables are typically stress tensors, but can also be strains or temperatures, whatever is needed to calculate the damage. The parameters $p_{L}$ depend on the point $M$ and the global behavior of the structure. For example, in the case of an elastic structure submitted to concentrated forces $\mathrm{F}$, under which its response can be considered quasi-static, the parameters $p_{L}$ can be seen as the $n_{F}$ localization tensors $\sigma_{L i}(M), i \in\left[1, n_{F}\right]$ such that the stress at point $M$ writes $\sigma(M, t)=\sum_{i} \sigma_{L i}(M) F_{i}(t)$.

The material damage function $D$ associates the history of local variables $\sigma$ and material parameters $p_{D}$ to the scalar variable of damage, $d$, in each point.

$$
\left[\begin{array}{c}
(\sigma(t))_{t \in[0, T]} \\
p_{D}
\end{array}\right] \stackrel{D}{\longrightarrow} d
$$

It is a local function that does not depend on the shape of the structure.

The structural damage function is then the composition of the localization function and the material damage function, written as $D \circ L$. Note that the explicit decomposition in two steps as previously described is not necessary. One may search directly for the function $D \circ L$ through the construction of a parametric meta-model, for example. For simpler notations the structural and material parameters $p_{L}$ and $p_{D}$ can be grouped in a single vector $p$.

$$
\left[\begin{array}{c}
(\mathrm{F}(t))_{t \in[0, T]} \\
p(M), M \in \Omega
\end{array}\right] \stackrel{D \circ L}{\longrightarrow} d
$$

The function $D \circ L$ describes the behavior (in terms of damage) of a family of structures among which can be found the structure that will indeed be designed. The space of parameters $p$ might be bounded in some directions by physical requirements. For example, if the Young modulus is involved, it should be positive. Moreover, this space might be restricted by the 
engineer, because he has some information about the structure to be designed like a choice of materials, fabrication process or general ideas about the shape of the structure. For example, the Young modulus of the material of an automotive structure is likely to be lesser than 210 $\mathrm{MPa}$, since steel is the stiffer material widely used in this field. However, theses assumptions have to be clearly stated because they restrict the applicability of the method.

Note that if two points $M_{1}$ and $M_{2}$ have the same parameter $p$, their damage will be equal. All the local information is contained in $p$. Consequently, it is equivalent to consider two points from a unique structure or two points from two different structures.

\subsection{Fatigue equivalent signal model}

The fatigue equivalent signal is a parametric model for a history of global loads $\mathrm{F}(t), t \in[0, T]$ suited for either simulation or physical tests purpose. The parameters $p_{e q}$ of the equivalent signal will be tuned to obtain the equivalence with the original signal. The notion of equivalence will be detailed further in this section.

The fatigue equivalent signal can be built as a simplification of the original signal; for example, by removing windows of the signal that do not (or little) damage the structure [17]. In this case, it is called a reduced signal and its parameters $p_{e q}$ are the indexes of the kept points, and the number of repetitions of the sequence of time points. This shortens the test duration, but does not solve the problem of controlling the test rigs. Moreover, this type of signal often remains too complex for simulation.

One therefore prefers a synthetic signal with few parameters. A popular choice for quasistatic design is block programs, consisting of a repeated sequence of several stages, each stage having a different waveform (usually sinus), mean value and amplitude, frequency and number of cycles. Other propositions, such as Gaussian processes or Markov Chains [13] can also be found in the literature. Gaussian signals are frequently used, especially for frequency response analysis and shaker experiments (see the so-called test tailoring method [10]).

It may be interesting to constrain some parameters of the equivalent signal a priori, in order to meet requirements that would not be included in the considered damage models or to confine the equivalent signal in the relevant domain of the damage model. For example, one may wish to keep the extreme values of the original signal to avoid exciting other (not described by the model) unwelcome mechanisms, such as plasticity. The frequency might also be limited in order to avoid any dynamic response if the model relies on a quasi-static assumption. Some constraints on the mixing sequence of small and large amplitudes can also reduce the sequence effect that is not taken into account in the simplest (and mostly used) Palgren-Miner accumulation law. In the case of an expected resonance of the structure, the extreme response spectrum is used to constrain the amplitude of the equivalent Gaussian signal [10].

\subsection{Strong formulation of the damage equivalence condition}

The general idea is to find values of the parameters of the equivalent signal, $p_{e q}$, such that in every point of any structure, the damage caused by the equivalent load is the same as the damage caused par the original load. The parameters which satisfy this condition are denoted $p_{e q}^{*}$. This condition writes :

$$
\text { Find } p_{e q}^{*} \text { such as } \forall p, \forall M, \quad D \circ L\left(\mathrm{~F}_{e q}\left(p_{e q}^{*}\right), p, M\right)=D \circ L(\mathrm{~F}, p, M)
$$

As was pointed out before, the damage depends on the point $M$ only through the parameter $p$ (which includes all the parameters for all the points), so that considering multiple structures 
comes down to considering multiple points, or more precisely, multiple $p$ :

$$
\text { Find } p_{e q}^{*} \text { such as } \forall p, \quad D \circ L\left(\mathrm{~F}_{e q}\left(p_{e q}^{*}\right), p\right)=D \circ L(\mathrm{~F}, p)
$$

with $p$ the parameters for any points of any structure in the scope of the design possibilities. Unfortunately this condition is almost never satisfied.

The one case when it is known to hold true is the 1D case described in section 1.3.1. The structure is elastic and quasi-static. The damage model is a combination of the linear accumulation law and a simple Basquin's model with a given material exponent $\beta$ for the damage model. It is then possible to find a constant amplitude, alternate signal equivalent in damage to the original one in every point of the structure.

With the same model, say that the structure is composed of two different materials, or suffer from two different damage mechanisms, so that you want the equivalent signal to be relevant for two different Basquin's exponents $\beta_{1}$ and $\beta_{2}$. It is still possible to find a solution to the equation 12 because fortunately the equivalent signal has two parameters. But this time, these parameters are completely determined by the two equations (5) given by $\beta_{1}$ and $\beta_{2}$ :

$$
F_{a e q}=\left(\frac{\sum_{j} F_{a}^{\beta_{1}}(j)}{\sum_{j} F_{a}^{\beta_{2}}(j)}\right)^{\frac{1}{\beta_{1}-\beta_{2}}} \text { and } \quad N_{e q}=\frac{\sum_{j} F_{a}^{\beta_{1}}(j)}{F_{a e q}^{\beta_{1}}}
$$

The user cannot choose the approximate number of cycles of the equivalent signal, which might be out of the relevant physical range of interest or reasonable test duration. Increasing the number of parameters of the equivalent signal can then give additional degrees of freedom to constrain the equivalent signal to stay in some given boundaries, or to take into account even more values of the Basquin's exponent $\beta$. A block-program with several (enough) different amplitudes can satisfy the equation 12 for a finite number of $\beta$.

\subsection{Weak formulation of the damage equivalence condition}

Now say that the precise material of the structure is not yet decided, or uncertain, so that this $\beta$ exponent is uncertain. It might take any value between $\beta_{1}$ and $\beta_{2}$. In this case, the fatigue equivalent signal would have to fulfill an infinite number or conditions, with a finite and preferably small number of parameters $p_{e q}$ (here, amplitudes and numbers of cycles), which in this case in not possible.

However, one can remark that first, the damage is calculated following a model with some amount of imprecision and second, that the fatigue life is highly scattered. Hence, a rigorous equivalence condition is not really needed and one can be content with an approximate condition.

This condition, here referred to as the "weak formulation of damage equivalence" ("weak" as "approximate") writes :

$$
p_{e q}^{*}=\underset{p_{e q}}{\operatorname{argmin}}\left\|D \circ L(\mathrm{~F}, p)-D \circ L\left(\mathrm{~F}_{e q}\left(p_{e q}\right), p\right)\right\|_{p}
$$

The notation $\|\bullet\|_{p}$ is a norm (to be defined) on the space of parameters and points of the structure. A simple choice is to take the maximum error over all the parameters; a quadratic norm (the variance of the error) could be another one (as in [13]). One could even imagine to weight the error with a probability density function of the unknown parameters.

The optimal parameters $p_{e q}^{*}$ are usually found thanks to some numerical minimization algorithm. It is usually necessary to discretize the parameter space in order to evaluate the 
error function. The error can be calculated a posteriori and some additional parameters $p_{e q}$ can be added if it is considered as too high.

This weak condition is less demanding than the strong formulation. However, in many cases, the variation of the damage error over "possible structures" (parameters $p$ ) is so large (nay infinite) that there is no way finding a fatigue equivalent signal with a reasonable (small) number of parameters. This case can happen when some parameters vary over a too large space. Moreover, a large size of the unknown parameter space leads to high computational times because the error function has to be evaluated on it.

\subsection{Strong formulation of the failure equivalence condition}

Following Thomas [8], reducing the space of "possible structures" can be done considering that the damage itself is of no interest to the design engineer, but only the failure behavior. The fatigue equivalent signal must provoke the same failure behavior as the original signal :

- the points that do not fail under the original signal should not fail either under the equivalent one;

- and reciprocally, the points that do fail under the original signal should also fail under the equivalent one.

This condition writes :

Find $p_{e q}^{*}$ such as $\forall p, \quad \operatorname{sign}\left(1-D \circ L\left(\mathrm{~F}_{e q}\left(p_{e q}^{*}\right), p\right)\right)=\operatorname{sign}(1-D \circ L(\mathrm{~F}, p))$

If $D \circ L(\mathrm{~F}, p)$ defines sufficiently regular isosurfaces in the neighborhood of 1 that the implicit function theorem applies in the unknown parameter space, the condition becomes:

$$
\text { Find } p_{e q}^{*} \text { such as } \forall p^{*} / D \circ L\left(\mathrm{~F}, p^{*}\right)=1, \quad D \circ L\left(\mathrm{~F}_{e q}\left(p_{e q}^{*}\right), p^{*}\right)=1
$$

\subsection{Weak formulation of the failure equivalence condition}

Here again, the strong equality is relaxed to get an easier condition to fulfill :

$$
p_{e q}^{*}=\underset{p_{e q}}{\operatorname{argmin}}\left\|1-D \circ L\left(\mathrm{~F}_{e q}\left(p_{e q}\right), p\right)\right\|_{p / D \circ L(\mathrm{~F}, p)=1}
$$

\section{Multi-input equivalent for an elastic quasi-static structure}

In this section, an application of the general approach exposed above is developed in the case of an elastic structure, undergoing infinitesimal strain and under quasi-static loading. The loading consists of a finite number of concentrated forces. It is an extension of Genêt's work [13] to the general case when no specific hypothesis is needed about the form of the local stress induced by each force [18].

The method is illustrated here in the case of only two input forces $F_{1}$ and $F_{2}$, and inducing a state of plane stress (cracks are supposed to initiate on the surface).

\subsection{Damage model}

The superposition principle applies :

$$
\sigma(M, t)=\sum_{i} \sigma_{L_{i}}(M) F_{i}(t)
$$


Here the localization stress tensors $\sigma_{L i}$ can take any form; they are not assumed to be proportional as in Genet's work. They are the unknown parameters of the structural damage function.

As in Genêt's thesis, Morel's model is here chosen to describe the fatigue damage. Only the main elements are stated here; for more details and physical arguments, see [13, 14]. Morel's model is based on a micro-macro approach. A scale transition model is proposed and the local damage is associated with the accumulated plastic strain on a critical glide system. The microscospic yield limit evolves in 3 phases (hardening,saturation, and softening) but here the saturation phase is supposed to be predominant so that the other phases are not taken into account.

In a spherical coordinate system defined by the angles $\theta, \phi, \psi$ a plane is defined by its normal unit vector $\mathrm{n}(\theta, \phi)$ and a direction on this plane by its unit vector $\mathrm{m}(\psi)$. The resolved shear stress on the glide system $(n, m)$ is then :

$$
\tau(\theta, \phi, \psi)=\operatorname{m} . \sigma \cdot n
$$

The critical plane $\left(\theta_{c}, \phi_{c}\right)$ maximizes the mean value (over directions $m$ ) of the variance of the resolved shear stress $\tau_{\text {rms }}^{2}$ :

$$
T_{r m s}=\max _{(\theta, \phi)} \sqrt{\int_{0}^{2 \pi} \tau_{r m s}^{2}(\theta, \phi, \psi) d \psi}
$$

The yield limit is computed as :

$$
\tau_{\text {lim }}=\frac{T_{r m s}}{C_{r m s}} \frac{-\alpha \sqrt{\pi} P_{m}+\beta \sqrt{\pi}}{\alpha \sqrt{\pi}+\frac{T_{r m s}}{P_{r m s}}} \frac{T_{r m s}}{P_{r m s}}
$$

with $P_{m}$ and $P_{r m s}$ the mean value and the standard deviation of the hydrostatic pressure, $\alpha$ and $\beta$ some material parameters (identical as those of the Dang Van criterion) and $C_{r m s}$ the maximum value over directions $m$ of the variance of the resolved shear stress $\tau_{r m s}^{2}$ on the critical plane:

$$
C_{r m s}=\max _{\psi} \tau_{r m s}\left(\theta_{c}, \phi_{c}, \psi\right)
$$

The damage then writes :

$$
d=\max _{\psi} \frac{\sum_{i}\left(\tau_{i}-\tau_{\text {lim }}\left(\theta_{c}, \phi_{c}, \psi\right)\right)_{+}}{2 q \tau_{\text {lim }}}
$$

with $(\bullet)_{+}$denoting the positive part, $q$ a material parameter, and $\tau_{i}$ the amplitude of the resolved shear stress on the glide system $\left(\theta_{c}, \phi_{c}, \psi\right)$ obtained by Rainflow counting on the variable $\tau$.

Note that any other criteria could be used as well. Here, this simplified version of Morel's criterion is chosen for its interesting balance between accuracy in case of complex loading and reasonable cost of evaluation thanks to the a priori calculation of the critical plane. The material parameters are given; they are not part of the unknown parameters of the structural damage function.

\subsection{Fatigue failure equivalency}

The weak formulation of the failure equivalence is applied. By combining the equations 18 and 23 , one can evaluate the structural damage function $d\left(F_{i}, \sigma_{L_{i}}\right)$. In order to simplify the notations, in the following, the localization tensors are simply noted $\sigma_{i}$. 


\subsubsection{Discretization of the unknown parameter space}

In a first step, the space of the unknown parameters $\sigma_{L}^{*}$ such that $d=1$ is searched. The space of stress tensors is parameterized as follows:

$$
\sigma_{\mathbf{1}}=K \cos \gamma\left(\begin{array}{ccc}
\sin \alpha_{1} & 0 & 0 \\
0 & \cos \alpha_{1} & 0 \\
0 & 0 & 0
\end{array}\right)_{\mathcal{B}_{1}} \quad, \quad \sigma_{2}=K \sin \gamma\left(\begin{array}{ccc}
\sin \alpha_{2} & 0 & 0 \\
0 & \cos \alpha_{2} & 0 \\
0 & 0 & 0
\end{array}\right)_{\mathcal{B}_{2}}
$$

with $R$ the rotation matrix between the principal directions basis $\mathcal{B}_{1}$ and $\mathcal{B}_{2}$ of $\sigma_{1}$ and $\sigma_{2}$ respectively:

$$
R=\left(\begin{array}{ccc}
\cos \alpha_{r o t} & -\sin \alpha_{r o t} & 0 \\
\sin \alpha_{r o t} & \cos \alpha_{r o t} & 0 \\
0 & 0 & 0
\end{array}\right)
$$

$K$ is a positive real number, $\alpha_{1}, \alpha_{2} \in[\pi / 4,3 \pi / 4], \alpha_{\text {rot }} \in[0, \pi / 2]$ (thanks to some symmetry of the damage function) and $\gamma \in[0,2 \pi]$. The following norm is defined over $\left(\sigma_{1}, \sigma_{2}\right)$ :

$$
\left\|\left(\sigma_{1}, \sigma_{2}\right)\right\|=\sqrt{\left\|\sigma_{1}\right\|^{2}+\left\|\sigma_{2}\right\|^{2}} \quad \text { with } \quad\|\sigma\|=\sqrt{\sigma: \sigma}
$$

so that, with the previously described parameterization : $\left\|\left(\sigma_{1}, \sigma_{2}\right)\right\|=K$. The space of unit stress tensors couples can then be explored by a variation on the angles $\alpha_{1}, \alpha_{2}, \alpha_{\text {rot }}$ and $\gamma$. Those angles evenly discretized for calculation purpose. The damage function is monotonic in $K$, hence there is only one $K^{*}$ such that $d\left(K^{*}\left\|\left(\sigma_{1}, \sigma_{2}\right)\right\|\right)=1$, easily found by dichotomy, for example.

\subsubsection{Fatigue equivalent model}

The equivalent signal is chosen as a block-program, each block a sinusoidal wave with 6 parameters : number of cycles $n$, mean values $F_{e q, m_{1}}$ and $F_{e q, m_{2}}$, amplitudes $F_{e q, a_{1}}$ and $F_{e q, a_{2}}$, and the phase $\varphi$ between the two input forces.

The procedure starts with a one-block signal. The optimal set of parameters for the equivalent signal is get thanks to standard minimization algorithms, with initial conditions given by the user. Several initial conditions are tested to avoid local minima. Using some more sophisticated minimization algorithm could probably improve this delicate process. The maximum error over the angles $\alpha_{1}, \alpha_{2}, \alpha_{\text {rot }}$ and $\gamma$ is checked and if it is superior to a given threshold, another block is added and the minimization procedure starts all over again. Blocks are thus added until the error criterion is reached. Usually few are required.

\subsection{Example}

The above described procedure is applied to a two-input signal recorded on a car component on a test track. The measured sequence is composed of nearly 6000 time points and is repeated 333 times. The component is made of steel with the material parameters $(\alpha=0.3$; $\beta=150 \mathrm{MPa} ; q=45000$ cycles ). The unknown parameter space (i.e. couples of localization tensors) is discretized as homogeneously as possible in 662 points, which can be interpreted as 662 structures. The threshold on damage is $1 \%$, thus a small value compared to the usual lifetime scatter for automotive steel sheets. The equivalent signal is shown on figure 1. Each block of sinus appears as an ellipse because of the phase between the two inputs. The phase allows to respect the desired amplitudes in all the directions of the input force space. The chosen criterion is not phase dependent but the material might be. In this case, some special constraints on the phase could be added. The quality of the equivalence can be checked on figure 2 . 


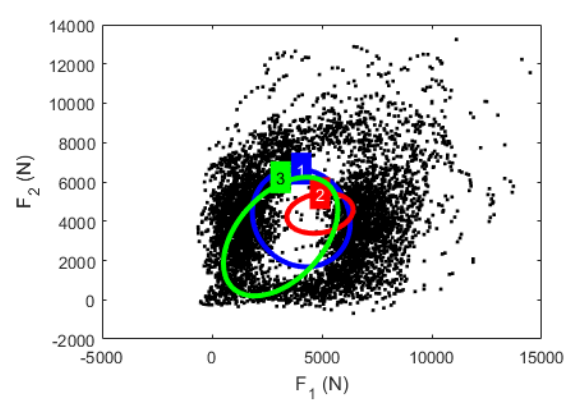

Figure 1. Two-input signal and 3 blocks equivalent signal. Block 1 is repeated 14518 times, block 219585 times and block 312113 times.

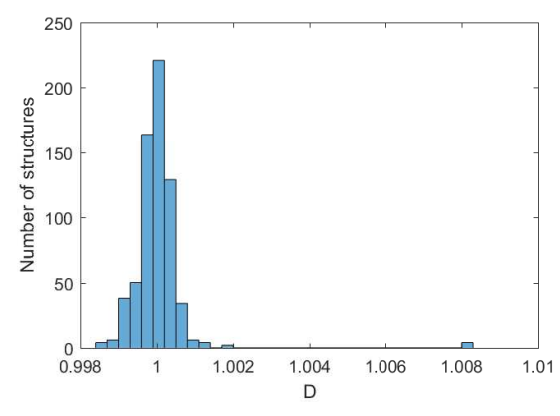

Figure 2. Distribution of the damage for the equivalent signal.

\section{Discussion}

The method proposed here needs a structural damage model precise enough to take into account the expected geometries and fatigue behavior of the structure, and yet simple enough to keep down the cost of an evaluation. The size of the input loads $n_{F}$ has a great influence on the duration and convergence of the minimization procedure. The substitution of the structural damage model by meta-models [19] could probably greatly improve the case of non-linear problems.

The size of the uncertain parameters $p$, in addition to (also) weighting on the calculation time, could over-constrain the problem, so that the expected simplification does not occur. This problem has not been encountered in the authors experience. Some knowledge about the structure is needed in order to restrain the size of $p$.

The question of a meaningful discretization and weighting of the parameter $p$ space is still an open question. Here, little attention has been paid to the weighting of the parameters (all possible values supposed to be equally relevant), but one could imagine describing the probability of each parameters (in the - unlikely?- situation when the design engineer has information to feed the method). The question of the discretization is more complex; it is mainly related to the regularity of the structural damage model over the parameter space.

Another point of interest is the way to choose an equivalent load form such that it has the relevant - and enough - degrees of freedom to satisfy the equivalence condition.

Many applications can be imagined and further developments are still to come.

\section{References}

[1] M. Lemaire, Structural Reliability, 1st edn. (Wiley-ISTE, 2009)

[2] C. Roux, X. Lorang, H. Maitournam, M.L. Nguyen-Tajan, Fatigue design of railway wheels: a probabilistic approach, Fatigue \& Fracture of Engineering Materials \& Structures 37, 1136 (2014), fFEMS-5471.R3

[3] P. Tibbits, Fatigue Load Equivalent to Distribution of Loads From the US Population, in ASME 2007 International Design Engineering Technical Conferences and Computers and Information in Engineering Conference (2007)

[4] G. Freebury, W. Musial, Determining Equivalent Damage Loading for Full-Scale Wind Turbine Blade Fatigue Tests February 2000 NREL / CP-500-27510, in 19th American Society of Mechanical Engineers (ASME) Wind Energy Symposium (Reno, Nevada, 2000) 
[5] B. Echard, N. Gayton, A. Bignonnet, A reliability analysis method for fatigue design, International Journal of Fatigue 59, 292 (2014)

[6] M. Matsuishi, T. Endo, Fatigue of metals subjected to varying stress, Tech. rep., Japan Society of Mechanical Engineers, Fukuoka, Japan (1968)

[7] H. Hendriks, B.H. Bulder, Fatigue equivalent load cycle method - a general method to compare the fatigue loading of different load spectrums, Tech. Rep. ECN-C-95-074, ECN (1995)

[8] J. Thomas, G. Perroud, A. Bignonnet, D. Monnet, in Fatigue Design and Reliability, edited by G. Marquis, J. Solin (Elsevier, 1999), Vol. 23 of European Structural Integrity Society, pp. $1-11$

[9] A. Halfpenny, Accelerated vibration testing based on fatigue damage spectra (2006)

[10] C. Lalanne, Mechanical Vibration E Shock: Fatigue damage, Mechanical Vibration \& Shock (Hermes Penton Science, 2002)

[11] F. Szmytka, A. Oudin, A reliability analysis method in thermomechanical fatigue design, International Journal of Fatigue 53, 82 (2013), proceedings of the 2nd International Workshop on Thermo-Mechanical Fatigue 2011

[12] M. Köhler, S. Jenne, K. Pötter, H. Zenner, Load assumption for fatigue design of structures and components (Springer-Verlag Berlin Heidelberg, 2017), translation from the German language edition: Zählverfahren und Lastannahme in der Betriebsfestigkeit.

[13] G. Genet, A statistical approach to multi-input equivalent fatigue loads for the durability of automotive structures, Ph.D. thesis, Chalmers University of Technology and Göteborg University (2006)

[14] F. Morel, A critical plane approach for life prediction of high cycle fatigue under multiaxial variable amplitude loading, International Journal of Fatigue 22, 101 (2000)

[15] C. Roux, X. Lorang, H. Maitournam, M.L. Nguyen-Tajan, B. Quesson, Multi-parameter fatigue equivalence loadings for specification applications, Procedia Engineering 66, 393 (2013), fatigue Design 2013, International Conference Proceedings

[16] B. Delattre, Analyse des chargements en service pour le dimensionnement fiabiliste à la fatigue polycyclique, in Colloque Mécamat - Fatigue des structures et des matériaux (Aussois, 2017)

[17] B. Weber, C. Montero, S. Bergamo, R. Rennert, A. Wünsche, S. Budano, I. Aranguren, Load spectrum lightening of fatigue tests data for time reduction of design validation Speefat, Tech. Rep. EUR 242006, European Commission - Research fund for coal and steel (2010)

[18] R. Jossic, Généralisation d'une méthode de construction d'équivalents fatigue multientrée, Master's thesis, École Centrale de Paris (2007)

[19] C. Farhat, A. Bos, P. Avery, C. Soize, Modeling and quantification of model-form uncertainties in eigenvalue computations using a stochastic reduced model, AIAA Journal 56, $1198(2018)$ 